\title{
Robotic resection of mediastinal goiter and ectopic thyroid
}

\author{
Luca Luzzi $^{1}$, Lisa De Leonibus ${ }^{1}$, Roberto Corzani ${ }^{1}$, Marco Ghisalberti ${ }^{1}$, Domenico Iemma ${ }^{2}$, \\ Carlo Enrico Ambrosini ${ }^{3}$, Pier Guido Ciabatti ${ }^{4}$, Piero Paladini ${ }^{1}$
}

${ }^{1}$ Thoracic Surgery Unit, Department of Medicine, Surgery and Neurosciences, University of Siena, Siena, Italy; ${ }^{2}$ Division of Anesthesiology, Siena
University Hospital, Siena, Italy; ${ }^{3}$ Endocrine and General Surgery Unit, S. Chiara University Hospital, Pisa, Italy; ${ }^{4}$ Otorhinolaringology Unit,
S. Donato Hospital, Arezzo, Italy
Contributions: (I) Conception and design: L Luzzi; (II) Administrative support: D Iemma; (III) Provision of study materials or patients: L De
Leonibus, CE Ambrosini; (IV) Collection and assembly of data: R Corzani, PG Ciabatti; (V) Data analysis and interpretation: P Paladini; (VI)
Manuscript writing: All authors; (VII) Final approval of manuscript: All authors.
Correspondence to: Luca Luzzi, MD, PhD. Thoracic Surgery Unit, Siena University Hospital, Viale Bracci 14, 53100 Siena, Italy. Email: dr.luca.luzzi@gmail.com.

\begin{abstract}
The last 10 years showed a wild diffusion of mini-invasive thoracic procedures for lung and mediastinum diseases. Mediastinal goiters, ectopic thyroids and forgotten goiters represent rare thoracic diseases, treated in the past by sternotomy, thoracotomy or combined cervicosternotomy. The evolution of robotic assisted thoracic surgery seems to offer a valid therapeutic option also in patients with thoracic and cervicothoracic thyroid correlate diseases. However some contraindications and technical aspects must be taken in account to achieve the best surgical results and patients satisfaction.
\end{abstract}

Keywords: Robotic thoracic surgery; mediastinal goiter; ectopic thyroid; forgotten goiter; cervical-thoracic surgical procedures

Received: 06 July 2018; Accepted: 08 November 2018; Published: 14 December 2018.

doi: $10.21037 /$ shc.2018.11.10

View this article at: http://dx.doi.org/10.21037/shc.2018.11.10

\section{Introduction}

The surgical management of thyroid diseases has been changed during the last two decades. The increased understanding of the endoscopic cervical anatomy, as well as the development of endoscopic instruments and the introduction of robotic surgery, pushed surgeons to offer minimally invasive thyroidectomy with small cervical incision or small incision in the transaxillary fold (1). The advantages of endoscopic over open surgery for thyroid diseases include a reduced rate of hyperesthesia and paresthesia of the neck and a highly improved cosmetic outcome.

On the same time, the minimally invasive surgery of the mediastinum, particularly the robotic surgery, offers a definitive therapeutic option in patients affected by intrathoracic ectopic thyroid tissue. In these patients, the advantage of a robotic approach is not only aesthetic, like for robotic standard thyroidectomy, but detrimental, avoiding the postoperative pain and functional respiratory impairment related to the median sternotomy. The robotic technique offers a 3-dimensional magnified view of the surgical field, a tremor free control and, most important, a hand-like articulated motion. The hand like motion let to achieve a precise and complete dissection from the thorax until the neck as demonstrated by the superior thymus horns dissection during radical thymectomy (2). Moreover there is the possibility to combine the thoracic and neck robotic procedures to resect thoracic and cervical lesions on the same time.

\section{Clinical presentation and diagnosis}

Thyroid disease can be divided into benign disease (multinodular goiter, multinodular toxic goiter and diffuse toxic goiter-Basedow disease) and malignant disease (differentiated and undifferentiated carcinomas). In case of benign normal functioning goiter the great majority of patients, even in presence of huge goiter, are asymptomatic. The symptomatic minority complains dysphagia and dyspnoea due to local tracheal and oesophageal compression 

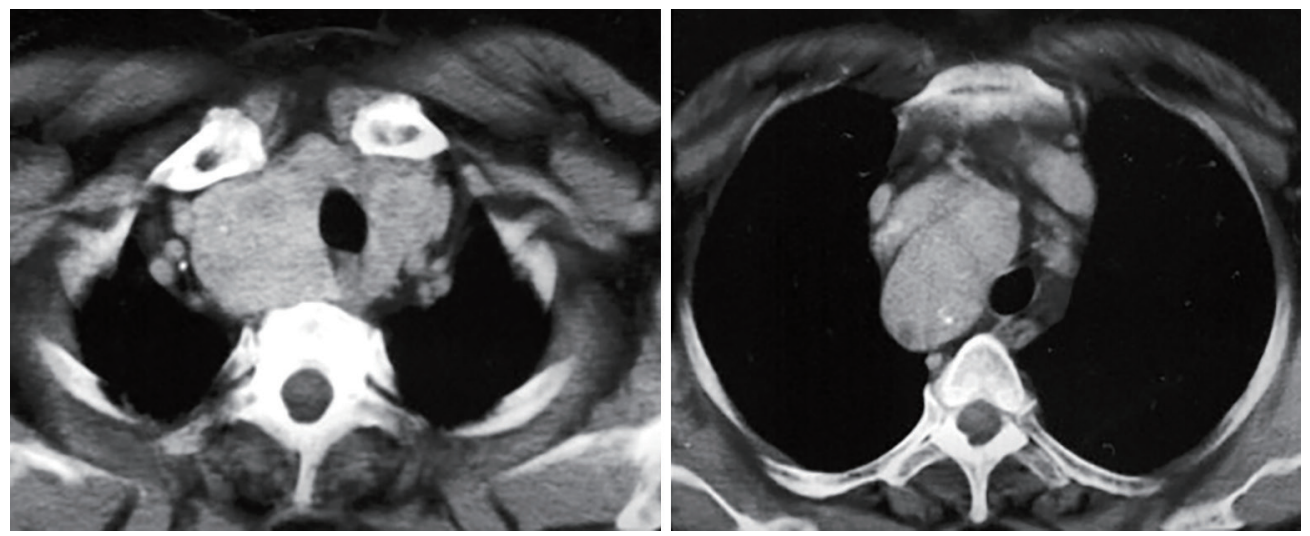

Figure 1 Typical cervicomediastinal goiter.

and deviation. Dysphonia is extremely rare. In case of hyperfunctioning disease, hyperthyroidism symptoms are present. Thyroid carcinomas are usually asymptomatic. Dysphonia due to recurrent laryngeal nerve infiltration by the primary tumor or by central compartment lymph node metastases is present only in locally advanced and aggressive carcinomas. After clinical examination and thyroid hormone blood assay ultrasound examination with fine needle aspiration of suspicious lesions is the first imaging study to be performed. Tc ${ }^{99}$ Scintigraphy and CT scan are second level studies, based on the clinical suspicion. The neck is not the exclusive localization of thyroid tissue. Three conditions, although rare, can be considered: the cervicomediastinal goiters, the ectopic thyroid gland and the so called "forgotten goiter". The cervicomediastinal goiter is generally struma located for at least $50 \%$ of its volume into the substernal space or into the mediastinum, in the pre-vascular or retro-vascular space, reaching at least the level of the aortic arch. For embryologic reasons, the cervicomediastinal goiters are mostly placed in the anterior or middle mediastinum (Figure 1), rarely in the posterior mediastinum (3). The majority of the affected patients are asymptomatic and the cervicomediastinal goiter is an occasional founding during normal assessment of a cervical goiter. In a small amount of these patients, respiratory symptoms can occur (due to tracheal compression), as well as vascular involvement (more often caused by neoplastic diseases) with compression of vessels and possible venous thrombosis.

The ectopic thyroid is a rare condition, due to a developmental abnormality during thyroid tissue migration from the floor of the primitive foregut to the neck. This is the reason why thyroid tissue can be found along the way of its descent, i.e. in the mediastinum, under the diaphragm or in other different neck sites (4). The majority of patients with ectopic thyroid are asymptomatic and for this reason the real incidence of the ectopic thyroid is unknown. Rarely patients complain obstructive symptoms, due to tracheal or vascular compression, or retrosternal pain; more often the symptoms are associated with chronic hypothyroidism, due to a disease of the ectopic gland that influences the orthotopic thyroid. Ectopic mediastinal thyroids are more frequently reported in the anterior mediastinum (Figure 2) or, as paraesophageal mass, in the posterior mediastinum; it is only rarely described in the paratracheal space, that is the more common localization of cervicomediastinal goiters instead. When the ectopic thyroid is developed in the anterior mediastinum on the left space, it can be mistaken for capsulated thymoma. In case of a homogenous vascularized mediastinal lesion, a simple and useful trick is to compare its Hounsfield Unit (HU) with the normal thyroid; if similar, ectopic thyroid can be suspected. The normal presence of iodine in the thyroid tissue gives an intrinsic characteristic improvement of density at CT scan without contrast that is specific of this tissue. On the other hand, the presence of an eggshell or ring calcifications are not specific, because they are commonly noticed in benign colloid nodules or papillary thyroid carcinomas (5), as well as in thymoma (6) or teratomas (Figure 3).

The last type of condition in which thyroid tissue can be found in the mediastinum is the forgotten goiter (Figure 4). In 1992 Massard (7) described seven cases of mediastinal thyroid tissue discovered in patients with history of total cervical thyroidectomy. This finding was called by Massard "goiter oublié" that means a forgotten goiter that may represent remnants of an incompletely resected 

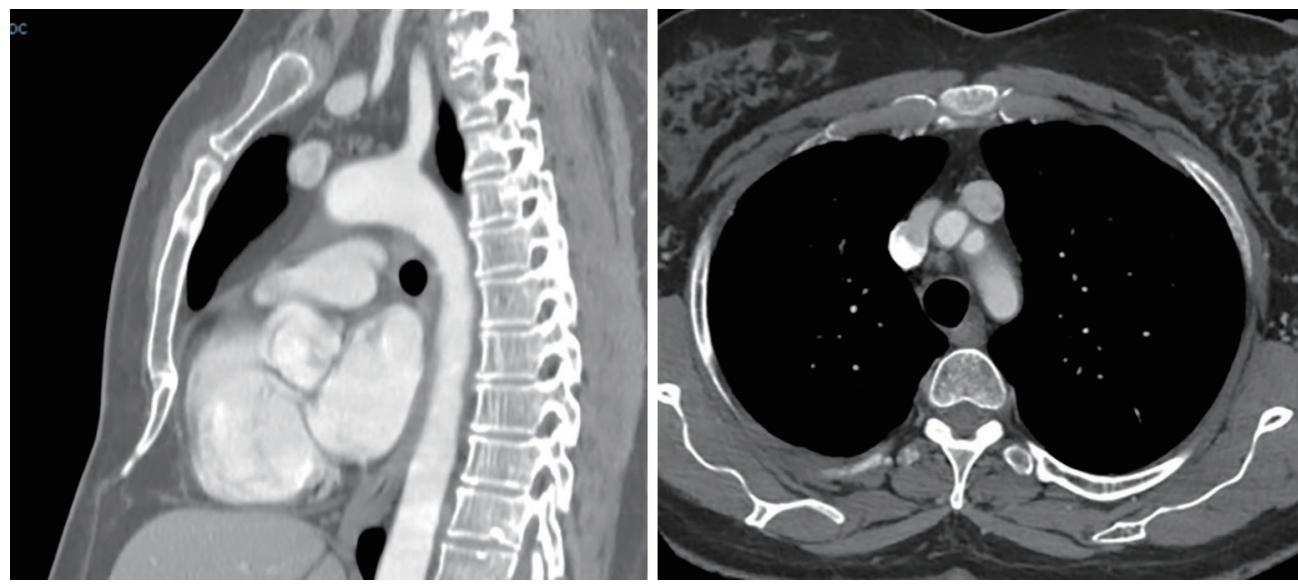

Figure 2 Ectopic thyroid in the anterior mediastinum.

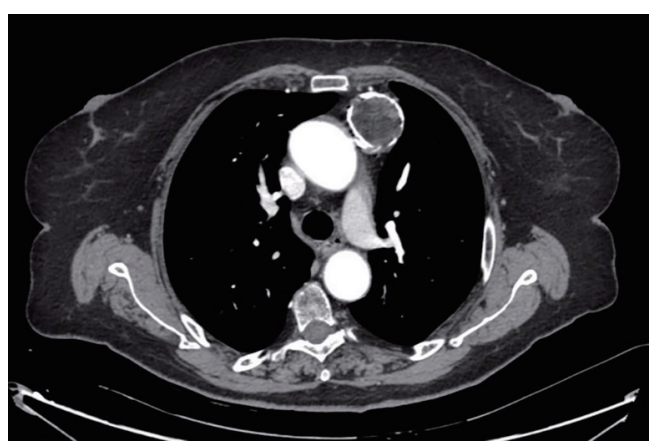

migrate inferiorly (8). The reviews published after the 1992 showed a range of latency from 1 to 39 years following the cervical thyroidectomy. The two major symptoms described are hormonal dysfunction or airway compression, however the majority of those patients are asymptomatic.

\section{Indications of mini-invasive robotic assisted surgery}

The indications for surgical treatment of cervical goiter and thyroid carcinomas are well standardized. Not all patients are suitable for mini-invasive surgery, such as those with a history of previous head-and-neck surgery or irradiation, uncontrolled thyrotoxicosis (in Graves-Basedow disease, the thyroid is rigid and difficult to manage, with a high risk to leave residual tissue behind) or lesions located in the dorsal thyroid area, especially in the region adjacent to the tracheoesophageal groove (because of the high risk of tracheal, esophagus, or recurrent laryngeal nerve injury). In addition, others contraindications to a mini-invasive procedure are locally advanced carcinomas infiltrating surrounding structures such as strap muscles, trachea, oesophagus or recurrent laryngeal nerve and presence of metastatic lymph nodes in central and lateral compartment (9). During the last years, a robotic assisted technique has been increasingly proposed instead of a mini-invasive endoscopic surgical approach. The advantage of robotic thyroidectomy is the absence of any cervical scar that in the Far East, especially in South Korea where this technique was born and developed, has a significant impact in patient's quality of life for cultural reasons (10). Nowadays, the two robot-assisted techniques proposed are the transaxillary, 


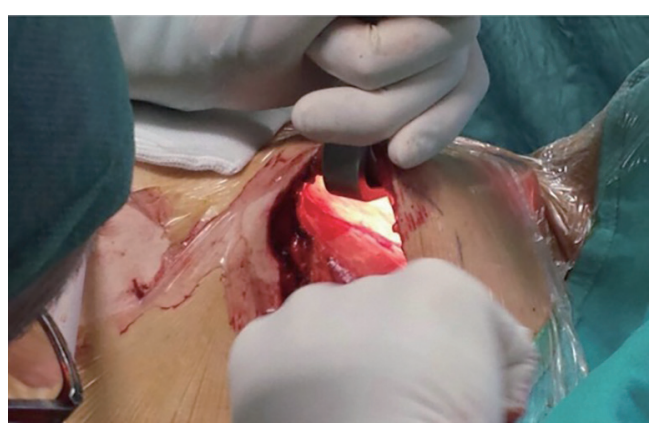

Figure 5 Tunnelization of the axillary access for cervical thyroidectomy.

most common and widely spread (11), and the transoral, less common and requiring a second axillary incision to retrieve large specimens (12). Despite these elegant and elaborate procedures, the real advantages of robotic cervical thyroidectomy are not confirmed by meta-analysis; it has been seen that the robotic approach needs a longer learning curve and longer operative time if compared to other non-robotic procedures, with similar complications rate. On the contrary, the opportunity to use a mini-invasive robotic approach for thoracic thyroid disease instead of traditional sternotomy, cervicosternotomy or thoracotomy, is potentially beneficial for the patients especially in case of "forgotten goiter" and ectopic thyroid tissue. Moreover it could be possible to perform combined robotic cervical and thoracic procedures for patients with synchronous thyroid and mediastinal disease using the same robot docking, saving time and reducing costs. According with the scientific literature and our experience, the indication to a single o combined robotic procedure has to be always discussed based on the type of thoracic goiter.

In case of cervicomediastinal goiter, the use of robot has been described only in a case in which the goiter was primarily approached through a cervicotomy and the robot assistance was used just to control the dissection of its thoracic portion (13). The thyroid tissue was taken out from the neck, as usually happen in the majority of the cases, and the support of the thoracic robotic procedure was minimal and probably not necessary. In fact, in the common practice, the thyroidectomy with en bloc removal of the intra-thoracic portion of the goiter by a cervical incision is appropriate in most of the cases. The mediastinal portion can be easily externalized by traction and digital dissection in a low vascularized plan as described by Di Crescenzo et al. (3).
The use of robotic surgery seems to be more appropriate in case of forgotten goiter or ectopic mediastinal goiter. In case of forgotten goiter, the patient has the cervical scar from the previous thyroidectomy and the majority of them have residual tissue laying in the retrosternal space; that leads the surgeon to repeat the cervical incision and resect the mediastinal tissue from the cervicotomy (14). In case of retrosternal deep forgotten goiter or thyroid tissue located in the middle mediastinum, the sternotomy was considered the treatment of choice in the past years (7). With the evolution of mini-invasive surgery and the robotic Da Vinci ${ }^{\mathrm{TM}}$ system, surgeons have a new opportunity to make a complete resection of these lesions with a prompt patients recovery and early discharge. The large experience acquired in robotic thymic surgery by thoracic surgeons during the last 10 years (15) can be easily transferred into the treatment of ectopic thyroid or forgotten goiter. In this case the minimal invasive robotic procedure from the thorax can avoid the problems related to the adhesions of previous neck surgery. In case of ectopic mediastinal thyroid, as well as mediastinal ectopic parathyroid, the indication of a robotic assisted resection is desirable.

\section{Surgical technique}

The robotic thyroidectomy starts usually from the side corresponding to the thyroid principal lesion. A 5 $\mathrm{cm}$ axillary incision following the posterior border of pectoralis major muscle is performed and a tunnel to the sternocleidomastoid muscle is prepared. The tunnelization is more accurate and safe by the help of a retractor equipped with an internal light (Figure 5). The sub-platysmal plane is thus developed between the skin and the pectoralis muscle and continued through the space between the sternal and the clavicle sternocleidomastoid heads. At this point, the thyroid Chung retractor is positioned to retract the strap muscle upward and the omohyoid muscle anteriorly, in order to permit an optimal exposition of the surgical field and the introduction of camera and two robotic arms (Figure 6). The tissues dissection follows the same steps occurring in standard open thyroidectomy and the procedure can be extended if cervical lymph node dissection is needed. The specimen is usually taken out from the axillary incision.

In case of thoracic ectopic goiter or forgotten goiter, the robotic resection should been scheduled based on the goiter position; this is important not only to decide the robot docking but also to determine the number of 


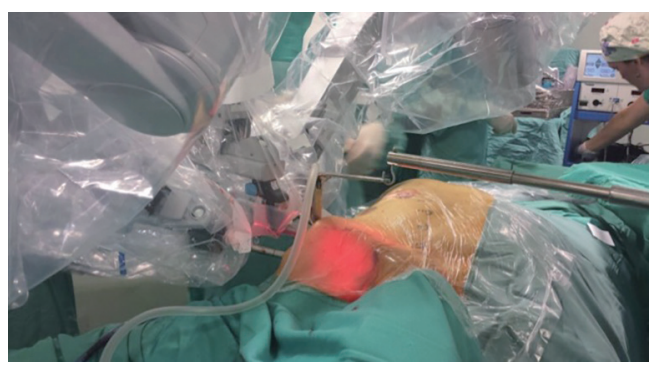

Figure 6 Insertion of the robotic arms in the axillary access.

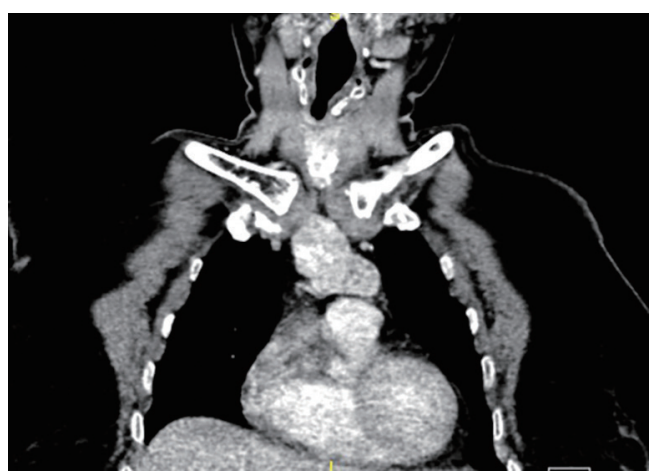

Figure 7 Anterior mediastinum goiter.

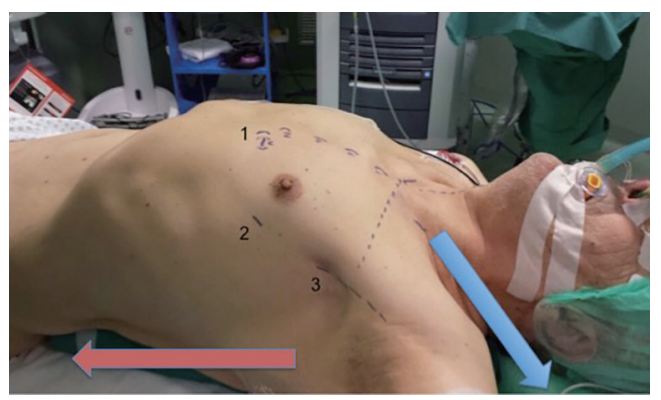

Figure 8 The three accesses utilized for standard anterior robotic approach for anterior mediastinal lesions. The arm is along the body (red arrow). In case of combined thoracocervical procedure the arm has to be extended (blue arrow) to allow the axillary access.

robotic arms to utilize, thus impacting on the procedure costs. In case of goiter found in the anterior mediastinum (Figure 7) three arms are sufficient to perform the entire procedure. The patient, intubated with a double-lumen endotracheal tube, is placed in supine position. Also in this case the side is chosen based on the hemi-thorax in which the goiter tends to be prominent. The right arm is positioned alongside the body to reduce the possibility of a shoulder injury. Then a roll is located under the thorax, in parallel to the spine, lifting the patient at $45^{\circ}$ in order to allow a little spread of the intercostal spaces and a major exposure of anterior mediastinum (Figure 8). Utilizing a Da Vinci Si the robot must be positioned contralateral to the access at the shoulder level; with the Da Vinci Xi, the kart position makes no difference. During surgery, a minimal controlled pneumothorax is achieved by $\mathrm{CO}_{2}$ inflation to improve the mediastinal space. The $\mathrm{CO}_{2}$ pressure is usually between 4 and $8 \mathrm{mmHg}$, rarely up to $10 \mathrm{mmHg}$ (set according to patient haemodynamic). The controlled pneumothorax facilitates the dissection, enlarging the space between sternum and pericardium for a better view of the contralateral mediastinum and cervical space. The thoracic approach is performed through three thoracoscopic accesses: a camera port $(12 \mathrm{~mm})$ in the 5 th intercostal space on anterior axillary line, an arm port $(8 \mathrm{~mm})$ in the 5 th intercostal space on hemi-clavicle line for the left arm and another one in the 3 rd intercostal space on anterior axillary line. In case of a preoperative diagnosis, the intervention is exclusively focused on the goiter resection. For the tissue dissection, we commonly use the harmonic scalpel but many surgeons describe excellent results also by using the monopolar spatula or the hook. The use of harmonic scalpel, despite not articulated, allows a blood free dissection with a good sealing even of the lymphatic vessels, potentially reducing the risk of chilothorax or, in general, the postoperative pleural effusion. In our experience about forgotten goiters or mediastinal ectopic goiters, we noticed a major vascularization of the fatty tissue around the goiter, associated with hypertrophic vessels; for this reason, the use of harmonic scalpel can strongly help the surgeon. In fact one of the most time-consuming problem in performing robotic surgery is the need of the operative field to be cleaned from blood by the assistant, forcing him to use one of the robotic accesses. In case of mediastinal anterior lesion suspected to be a goiter but not confirmed, the surgical resection addressed should be a complete thymectomy because of the chance of a thymoma. In any case, the specimen must be placed in the retrieval bag and removed from one of the access; we usually use the axillary access for aesthetic reasons, leaving the drainage in the camera-port incision. Same authors described rare cases of forgotten goiter or ectopic goiter in the middle and posterior mediastinum. In these cases, the best strategy is to put the patient in the lateral position. This allows a better view of 
the posterior and middle mediastinum, but it requires the activation of the forth robotic arm, in order to manage the lung tissue that tends to lie in the costovertebral space when excluded from ventilation. The ports setup is performed with four robotic accesses (Figure 9). Three accesses are performed in the 9 th intercostal space, approximately $8 \mathrm{~cm}$ between each other, starting from the camera port $(12 \mathrm{~mm})$ on the middle axillary line and continuing posteriorly for the second and third arm placement. The fourth arm is located in the 5th intercostal space on anterior axillary line, usually equipped with the tip-up forceps for the lung management. During the last 2 years, we performed two cases of

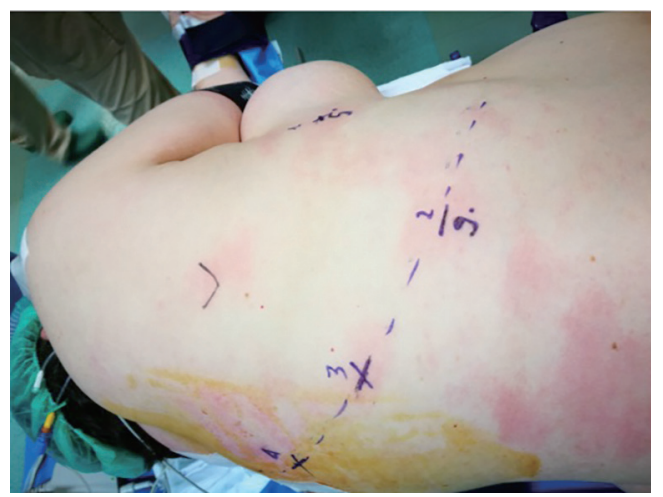

Figure 9 In case of Goiter localized in the posterior or middle mediastinum we suggest a posterolateral position. The trocars are placed in the $9^{\text {th }}$ and $5^{\text {th }}$ spaces with the camera starting in position 2 . This docking allows to explore all posterior mediastinum from the apex to the diaphragm. combined cervical and thoracic robotic procedure (16) in patients with synchronous mediastinal lesion and goiter (Figure 10). In case of combined intervention, it is necessary to start with the thoracic resection to maintain the $\mathrm{CO}_{2}$-induced pneumothorax. The axillary incision for robotic thyroidectomy is performed enlarging the skin incision of the axillary port used for robotic thoracic procedure, no more able to contain the $\mathrm{CO}_{2}$. To permit a simultaneous robot docking for the two homo-lateral procedures, the patient arm, corresponding to the axillary access, needs to be exposed over the head as shown in Figure 9. In this way it is possible to shift from the thoracic to the cervical procedure just with a simple $30^{\circ}$ rotation of the robot (Figure 11) in case of $\mathrm{Da}$ Vinci $\mathrm{Si}$, or a simple arms repositioning using the new Da Vinci Xi.

\section{Conclusions}

If the robotic trans-axillary or trans-oral robotic thyroidectomy is not proved to achieve superior advantages to mini-invasive or traditional cervical procedure, for thoracic ectopic or forgotten goiters the possibility to avoid a sternotomy or cervicosternotomy is detrimental for patient recovery. In these cases, especially when a preoperative diagnosis is available, the opportunity of a video-assisted thoracic surgery (VATS) resection could be considered. However, especially for anterior mediastinal goiter, a complete thymectomy should be achieved in the suspect of a localized capsulate thymoma. For this reason the robotic assisted procedure is more appropriate in the
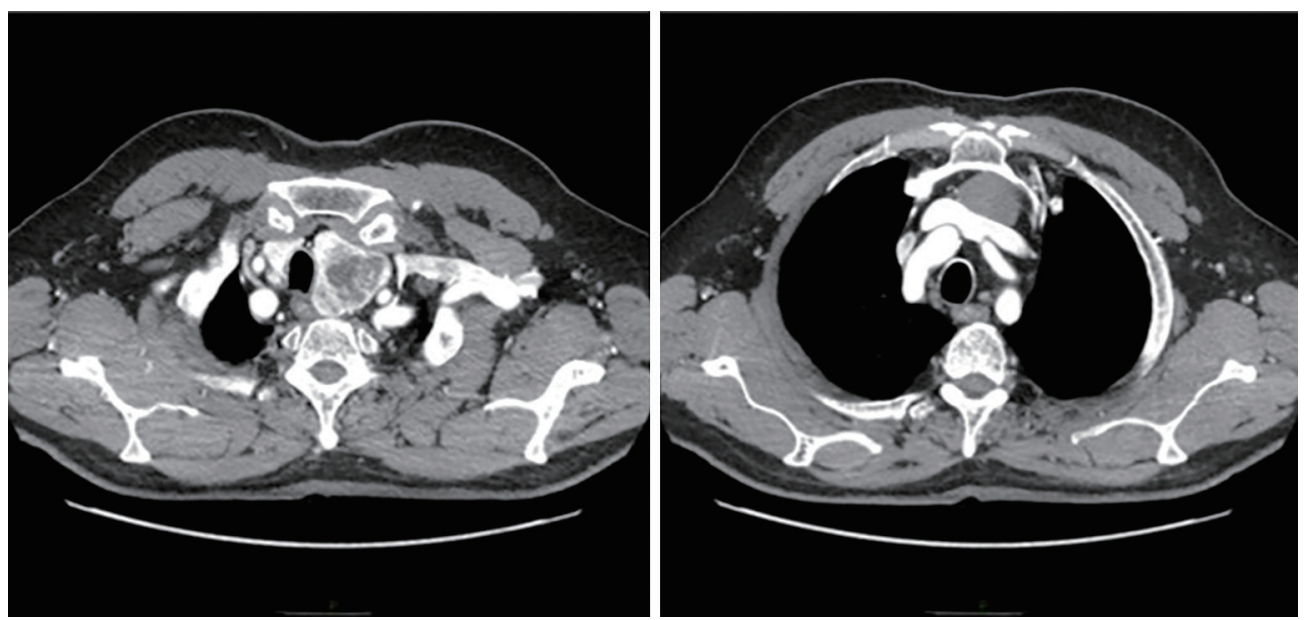

Figure 10 The CT scan of a patient affected by a cervical goiter and an anterior mediastinal lesion associated with miastenia gravis who received a total thymectomy and a complete thyroidectomy by a combined robotic assisted procedure. 


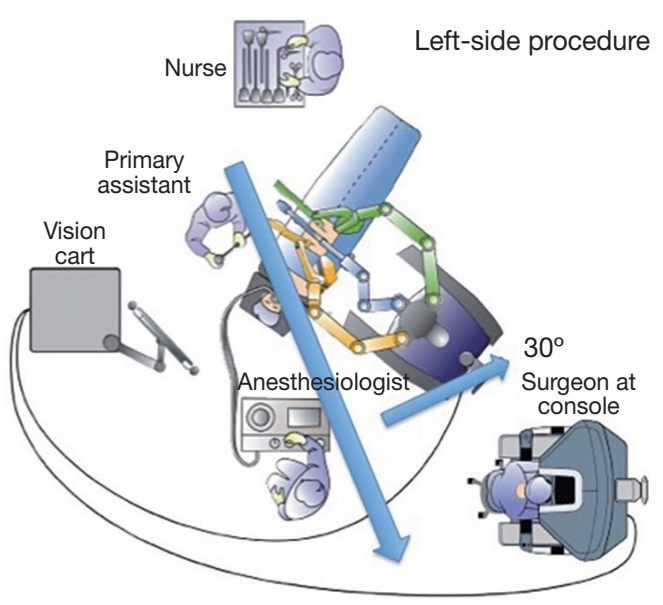

Figure 11 The $30^{\circ}$ movement of the robot Da Vinci Si allows the axillary access after the robotic thymectomy [with the permission of Luzzi et al. (16)].

effort to obtain a complete radical resection, due to the more precise dissection, reducing the risk of blooding.

\section{Acknowledgments}

Funding: None.

\section{Footnote}

Provenance and Peer Review: This article was commissioned by the Guest Editor (Giuseppe Marulli) for the series "Robotic Mediastinal Surgery" published in Shanghai Chest. The article has undergone external peer review.

Conflicts of Interest: All authors have completed the ICMJE uniform disclosure form (available at http://dx.doi. org/10.21037/shc.2018.11.10). The series "Robotic Mediastinal Surgery" was commissioned by the editorial office without any funding or sponsorship. The authors have no other conflicts of interest to declare.

Ethical Statement: The authors are accountable for all aspects of the work in ensuring that questions related to the accuracy or integrity of any part of the work are appropriately investigated and resolved.

Open Access Statement: This is an Open Access article distributed in accordance with the Creative Commons Attribution-NonCommercial-NoDerivs 4.0 International
License (CC BY-NC-ND 4.0), which permits the noncommercial replication and distribution of the article with the strict proviso that no changes or edits are made and the original work is properly cited (including links to both the formal publication through the relevant DOI and the license). See: https://creativecommons.org/licenses/by-nc-nd/4.0/.

\section{References}

1. Kandil E, Hammad AY, Walvekar RR, et al. Robotic Thyroidectomy versus non robotic approaches: a metaanalysis examining surgical outcomes. Surg Innov 2016;23:317-25.

2. Marulli G, Comacchio GM, Schiavon M, et al. Comparing robotic and trans-sternal thymectomy for early-stage thymoma: a propensity score-matching study. Eur J Cardiothorac Surg 2018;54:579-84.

3. Di Crescenzo V, Vitale M, Valvano L, et al. Surgical management of cervico-mediastinal goiters: Our experience and review of the literature. Int J Surg 2016;28 Suppl 1:S47-53.

4. Ibrahim NA, Fadeyibi IO. Ectopic Thyroid: etiology, pathology and management. Hormones 2011;10:261-9.

5. Yuzbasioglu MF, Ozkaya M, Ezberci F, et al. Eggshell calcification after intrathyroidal hemorrhage of retrosternal thyroid. Cases J 2008;1:11.

6. Harris K, Elsayegh D, Azab B, et al. Thymoma calcification: is it clinical meaningful? World J Surg Oncol 2011;9:95.

7. Massard G, Wihlm JM, Jeung MY, et al. Forgotten mediastinal goiter: seven cases. Ann Chir 1992;46:770-3.

8. Calò PG, Tatti A, Medas F, et al. Forgotten goiter: our experience and a review of the literature Ann Ital Chir 2012;83:487-90.

9. Lee J, Chung WY. Robotic surgery for thyroid disease. Eur Thyroid J 2013;2:93-101

10. Aidan P, Arora A, Lorincz B, et al. Robotic Thyroid surgery: Current Perspectives and future considerations. ORL J Otorhinolaryngol Relat Spec 2018;80:186-94.

11. Sun GH, Peres L, Pynnonen MA, et al. Systematic review and meta-analysis of robotic vs conventional thyroidectomy approaches for thyroid disease. Otolaryngol Head Neck Surg 2014;150:520-32.

12. Russell JO, Clark J, Noureldine SI, et al. Transoral thyroidectomy and parathyroidectomy - A North American series of robotic and endoscopic transoral approaches to the central neck. Oral Oncol 2017;71:75-80.

13. Wang S, Xu S, Liu B. Resection of huge retrosternal goiter through a novel combined cervical and robot-assisted approach. Artif Organs 2014;38:431-3. 
14. Patel KM, Parsons CC. Forgotten Goiter: diagnosis and management. A case report and literature review. Int J Surg Case Rep 2016;27:192-4.

15. Marulli G, Maessen J, Melfi F, et al. Multi-institutional European experience of robotic thymectomy for thymoma.
Ann Cardiothorac Surg 2016;5:18-25.

16. Luzzi L, Corzani R, Burali G, et al. First case of combined robot-assisted Thymectomy and transaxillary thyroidectomy: technique and robot-docking optimization. J Robot Surg 2017;11:239-41.

doi: $10.21037 /$ shc. 2018.11 .10

Cite this article as: Luzzi L, De Leonibus L, Corzani R, Ghisalberti M, Iemma D, Ambrosini CE, Ciabatti PG, Paladini P. Robotic resection of mediastinal goiter and ectopic thyroid. Shanghai Chest 2018;2:94. 